\title{
Risk factors for hyperglycemia in pregnancy in the DALI study differ by period of pregnancy and OGTT time point
}

\author{
Lilian C Mendoza, * , Jürgen Harreiter2,*, David Simmons ${ }^{3,4}$, Gernot Desoye ${ }^{5}$, J M Adelantado6, Fabiola Juarez ${ }^{6}$ \\ Ana Chico 1,6,7, Roland Devlieger ${ }^{8,9}$, Andre van Assche ${ }^{8,9}$, Sander Galjaard ${ }^{8,9}$, Peter Damm ${ }^{10,11,}$ \\ Elisabeth R Mathiesen ${ }^{10,11}$, Dorte M Jensen ${ }^{12,13}$, Lise Lotte T Andersen ${ }^{12,13}$, Mette Tanvig ${ }^{12,13}$, \\ Annunziata Lapolla ${ }^{14}$, Maria G Dalfra ${ }^{14}$, Alessandra Bertolotto ${ }^{15}$, Urszula Mantaj ${ }^{16}$, Ewa Wender-Ozegowska ${ }^{16}$, \\ Agnieszka Zawiejska ${ }^{16}$, David Hill ${ }^{17}$, Judith G Jelsma ${ }^{18}$, Frank J Snoek ${ }^{19}$, Mireille N M van Poppel ${ }^{18,20}$, \\ Christof Worda21, Dagmar Bancher-Todesca ${ }^{21}$, Alexandra Kautzky-Willer,22, Fidelma P Dunne ${ }^{23}$ and \\ Rosa Corcoy ${ }^{1,6,7}$ on behalf of the DALI Core Investigator Group
}

1Department of Medicine, Universitat Autònoma de Barcelona, Barcelona, Spain, ²Division of Endocrinology, Department of Medicine III, Gender Medicine Unit, Medical University of Vienna, Vienna, Austria, Institute of Metabolic Science, Addenbrookes Hospital, Cambridge, UK, ${ }^{4}$ Macarthur Clinical School, Western Sydney University, Sydney, Australia, ${ }^{5}$ Department of Obstetrics and Gynecology، Medizinische Universitaet Graz, Graz, Austria, ${ }^{6}$ Institut de Recerca de I'Hospital de la Santa Creu i Sant Pau, Barcelona, Spain, ${ }^{7} \mathrm{CIBER}$ Bioengineering, Biomaterials and Nanotechnology, Instituto de Salud Carlos III, Zaragoza, Spain, ${ }^{8} \mathrm{KU}$ Leuven, Department of Development and Regeneration: Pregnancy, Fetus and Neonate, Leuven, Belgium, ${ }^{9}$ Gynaecology and Obstetrics, University Hospitals Leuven, Leuven, Belgium, ${ }^{10}$ Center for Pregnant Women with Diabetes, Departments of Endocrinology and Obstetrics, Rigshospitalet, Copenhagen, Denmark, ${ }^{11}$ Institute of Clinical Medicine, Faculty of Health and Medical Sciences, University of Copenhagen,

Copenhagen, Denmark, ${ }^{12}$ Departments of Endocrinology, Gynaecology and Obstetrics, Odense University Hospital, Odense, Denmark, ${ }^{13}$ Department of Clinical Research, Faculty of Health Science, University of Southern Denmark, Odense, Denmark, ${ }^{14}$ Universita Degli Studi di Padova, Padua, Italy, ${ }^{15}$ Azienda Ospedaliero-Universitaria Pisana, Pisa, Italy, ${ }^{16}$ Division of Reproduction, Medical Faculty I, Poznan University of Medical Sciences, Poznan, Poland, ${ }^{17}$ Recherche en Santé Lawson SA, St Gallen, Switzerland, ${ }^{18}$ Department of Public and Occupational Health, Amsterdam Public Health Research Institute, VU University Medical Centre, Amsterdam, the Netherlands, ${ }^{19}$ Department of Medical Psychology, VU University Medical Centre and Academic Medical Centre, Amsterdam, the Netherlands, ${ }^{20}$ Institute of Sport Science, University of Graz, Graz, Austria, ${ }^{21}$ Division of Obstetrics and Feto-Maternal Medicine, Department of Obstetrics and Gynecology, Medical University of Vienna, Vienna, Austria, ${ }^{22}$ Gender Medicine Institute, Gars am Kamp, Austria, and ${ }^{23}$ National University of Ireland, Galway, Ireland

\begin{abstract}
Objective: Risk factors are widely used to identify women at risk for gestational diabetes mellitus (GDM) without clear distinction by pregnancy period or oral glucose tolerance test (OGTT) time points. We aimed to assess the clinical risk factors for Hyperglycemia in pregnancy (HiP) differentiating by these two aspects.

Design and methods: Nine hundred seventy-one overweight/obese pregnant women, enrolled in the DALI study for preventing GDM. OGTTs were performed at $\leq 19+6,24-28$ and 35-37 weeks (IADPSG/WHO2013 criteria). Women with GDM or overt diabetes at one time point did not proceed to further OGTTs. Potential independent variables included baseline maternal and current pregnancy characteristics. Statistical analysis: Multivariate logistic regression. Results: Clinical characteristics independently associated with GDM/overt diabetes were at $\leq 19+6$ weeks, previous abnormal glucose tolerance (odds ratio (OR): $3.11 ; 95 \% \mathrm{Cl}: 1.41-6.85)$, previous GDM (OR: $2.22 ; 95 \% \mathrm{Cl}: 1.20-4.11$ ), neck circumference (NC) (OR: 1.58; $95 \% \mathrm{Cl}: 1.06-2.36$ for the upper tertile), resting heart rate (RHR, OR: $1.99 ; 95 \% \mathrm{Cl}$ : 1.31-3.00 for the upper tertile) and recruitment site; at 24-28 weeks, previous stillbirth (OR: $2.92 ; 95 \%$ Cl: 1.18-7.22), RHR (OR: 3.32; 95\% Cl: 1.70-6.49 for the upper tertile) and recruitment site; at 35-37 weeks, maternal height (OR: $0.41 ; 95 \% \mathrm{Cl}: 0.20-0.87$ for upper tertile). Clinical characteristics independently associated with GDM/overt diabetes differed by OGTT time point (e.g. at $\leq 19+6$ weeks, NC was associated with abnormal fasting but not postchallenge glucose).
\end{abstract}

(C) 2018 European Society of Endocrinology Printed in Great Britain
Published by Bioscientifica Ltd. 
Conclusion: In this population, most clinical characteristics associated with GDM/overt diabetes were non-modifiable and differed by pregnancy period and OGTT time point. The identified risk factors can help define the target population for future intervention trials.

\section{Introduction}

Hyperglycemia in pregnancy (either gestational diabetes mellitus (GDM) or overt diabetes) is associated with adverse pregnancy outcomes. GDM, currently defined by the American Diabetes Association (ADA) as diabetes diagnosed in the second or third trimester of pregnancy that is not clearly overt diabetes (1) is mainly associated with macrosomia and perinatal complications related to excessive fetal growth (shoulder dystocia, cesarean section), as well as neonatal and maternal morbidity (2).

The Hyperglycemia And Pregnancy Outcomes (HAPO) study showed that risk of adverse perinatal outcomes increased as a function of maternal glycemia, even within ranges considered normal for pregnancy (3). Recent data, using the IADPSG/WHO2013 diagnostic criteria (4), reveal that GDM affects 9-25\% of all pregnancies (5).

As GDM, or even overt diabetes in pregnancy, rarely presents with symptoms, diagnosis is performed by screening, and clinical risk factors are used to identify it in early pregnancy $(1,6,7)$ or at $24-28$ weeks $(7)$. However, the possibility of differences in risk factors for early vs later screening have not been addressed in the literature, probably because screening is usually selective in early pregnancy $(6,7)$. Selective screening is mostly based on weight status, since excess weight is one of the major identified risk factors for GDM (8), with overweight/obese women being 2-5.6 times more likely to develop the condition (9). However, not all overweight/obese women develop GDM, and being able to determine women at increased risk within this group could help optimize screening strategies. In turn, the contribution of different oral glucose tolerance test (OGTT) time points to GDM diagnosis significantly differed across centers in the HAPO study (5), and the reasons are not completely understood.

The main aim of the DALI study was to evaluate the efficiency of lifestyle intervention and vitamin D supplementation in reducing gestational weight gain, fasting plasma glucose (FPG) and improving insulin sensitivity measured with the homeostasis model assessment. The specific aim of this analysis was to assess if clinical factors associated with GDM/overt diabetes differ by pregnancy period and OGTT time point.

\section{Subjects and methods}

\section{Study population}

Nine hundred eighty-four women were recruited to participate in the DALI study for the prevention of GDM; only women with OGTT data are included in the current analysis. The DALI study was a multicenter randomized controlled trial testing approaches for the reduction in the risk of GDM, that included: (1) a pilot trial, (2) the DALI lifestyle study comparing healthy eating (HE), physical activity (PA) and combined healthy eating and physical activity (HE\&PA) interventions with a control group and (3) the DALI vitamin D study comparing vitamin D supplementation with and without HE\&PA vs placebo.

Eligibility criteria were prepregnancy BMI $\geq 29 \mathrm{~kg} /$ $\mathrm{m}^{2}, \leq 19+6$ weeks of gestation, singleton pregnancy and age $\geq 18$ years. Gestational age was estimated using selfreported date of last menstrual period, corrected after ultrasound if required. Exclusion criteria were being unable to walk at least $100 \mathrm{~m}$ safely or to speak the language of the recruitment site, complex diet requirements and chronic medical or psychiatric conditions. Women were recruited in 9 European countries at 11 different sites. Consent was obtained from each participant after full explanation of the purpose and nature of all procedures used in the study. Women diagnosed with overt diabetes or GDM with IADPSG/WHO 2013 criteria $\leq 19+6$ weeks of gestation were excluded from the randomized trials, but included in the analyses presented here.

The study was approved by the relevant ethical committees (NRES Committee East of England - Norfolk: 11/EE/0221; Medical University Poznan: 1165/12; UZ KU Leuven: ML7625; VUmc Amsterdam: 2012/400; Hospital De La Santa Creu i Sant Pau Barcelona 13/006 (OBS); Medical University Vienna: 2022/2012 - 1369/2013; Region Hovedstaden Copenhagen: H-4-2013-005; Province of Padua: $4201 \AA$ 11; Galway University Hospitals: 7/12).

\section{Data collection}

Data from participating women were collected at three time points: baseline $(\leq 19+6$ weeks of pregnancy), 
at 24-28 weeks and at 35-37 weeks. At these time points, blood samples were collected, anthropometric measurements were performed and participants were asked to complete different questionnaires.

At each time point, women undertook a standardized, $75 \mathrm{~g}$ OGTT in sitting position after a 10-h fast, unless GDM or overt diabetes had been diagnosed in an earlier assessment. IADPSG/WH2013 criteria were used (4). Blood samples were analyzed at local and central laboratories, with local results being used for clinical management. For the current analysis, central laboratory values were used (local data used when central data were unavailable).

Socio-demographic information was collected at baseline, including maternal age, ethnicity, education, employment and marital status. Anamnesis included family history of diabetes in first-degree relatives, active smoking, polycystic ovarian syndrome (PCOS), chronic hypertension, previous abnormal glucose tolerance (AGT) and obstetric history (previous pregnancies, stillbirth, macrosomia, congenital anomalies, previous GDM).

Physical examination at each visit included measurements of resting heart rate (RHR), and anthropometrics including height, weight, BMI and neck circumference (NC). RHR was measured with an electronic blood pressure monitor after $5 \mathrm{~min}$ of rest (average value of two measurements taken 1-min apart). Prepregnancy weight was based on self-report, using the earliest antenatal weight when prepregnancy weight was not known. Height was measured at baseline with a stadiometer. Height measurement and prepregnancy weight were used to calculate BMI $\left(\mathrm{kg} / \mathrm{m}^{2}\right)$, to determine inclusion criteria. In the next time points, women were weighed on calibrated electronic scales and BMI was calculated in each visit. NC, a central adiposity index, was considered because of its association with various cardiovascular risk factors (10), and insulin resistance (11), its easy measurement (12) and independent association with pregnancy-induced hypertension and preeclampsia (13). NC was measured in a standing relaxed upright position between mid-cervical spine and mid-anterior neck, to within $1 \mathrm{~mm}$ (12). Taking into account information in the literature, the season during which the OGTT was performed (14) and newborn sex (15) was also addressed.

Outcome variable: GDM or overt diabetes according to IADPSG/WHO2013 criteria (FPG $\geq 5.1 \mathrm{mmol} / \mathrm{L}$ and/or 1-h plasma glucose $(\mathrm{PG}) \geq 10 \mathrm{mmol} / \mathrm{L}$ and/or $2 \mathrm{~h} \mathrm{PG}$ $\geq 8.5 \mathrm{mmol} / \mathrm{L}$ ).

Potential Hyperglycemia in pregnancy (HiP) risk factors studied were first-degree family history of diabetes, maternal age, ethnicity, education, employment, marital status, active smoking, chronic hypertension, PCOS, previous AGT, previous pregnancies, previous stillbirth, previous macrosomia, previous congenital anomalies, previous GDM, maternal weight and BMI at different pregnancy periods, height, NC, RHR, recruitment site, newborn sex, season when OGTT was performed and (at 24-28 and 35-37 weeks) study intervention.

\section{DALI interventions}

HE and PA interventions used motivational interviewing method. The HE intervention aimed to promote a diet lower in simple and complex carbohydrate, lower fat, limited intake of total calories while keeping protein intake and increasing fiber. The PA intervention was in line with American College of Obstetricians and Gynecologists (ACOG) guidelines and promoted both aerobic and resistance PA. The vitamin D intervention used a supplementation of 1600 IU/day of vitamin D3 on top of pregnancy vitamins taken by the women (16).

The trial registration number is ISRCTN70595832 and the detailed protocol has already been published (16).

\section{Statistical analyses}

Categorical data are presented as counts and percentages, and continuous data as mean and standard deviation or as median and interquartile range for data not normally distributed.

Overall prevalence of GDM/overt diabetes was calculated as the rate of women with a GDM/overt diabetes diagnosis among those with glucose data. The overall prevalence per protocol was calculated as the rate of women with a GDM/overt diabetes diagnosis among those completely adhering to the study protocol (all expected OGTTs performed by the last day of pregnancy).

First, a bivariate logistic regression was performed for potential GDM/overt diabetes risk factors at each pregnancy period and OGTT time point. Factors with a two-sided $P$ value $<0.10$ were included in a multivariate backward logistic regression model to identify independent variables and calculate the corresponding OR. Barcelona was empirically chosen as the reference site. The components of the DALI intervention (HE, PA, vitamin D) were dealt with as different variables. Significance was defined as a two-sided $P<0.05$. The ORs for continuous independent variables were turned into tertiles to assess their relevance in clinical terms.

To test the performance of the models obtained, receiver-operating characteristic curves (ROC) were 
performed, and the area under the ROC curve (AUROC) was calculated for each pregnancy period.

Two different sensitivity analyses were performed.

The first sensitivity analysis was performed at each pregnancy period using prepregnancy weight and BMI and standardized changes in weight ( $\Delta$ weight) and BMI ( $\Delta$ BMI) from prepregnancy instead of particular measurements at each point. $\Delta$ weight was defined as the standardized difference between maternal weight at 24-28 or 35-37 weeks and prepregnancy weight. $\Delta$ BMI was defined as the standardized difference between BMI at 24-28 or 35-37 weeks and prepregnancy BMI.

A second sensitivity analysis was performed using mixed-effects logistic regression model with center as a group variable.

All statistical analyses were performed with the statistical package IBM SPSS Statistics (IBM Corp, version 20.0), except for the mixed-effects logistic regression that was performed with STATA.

\section{Results}

Of the 984 women recruited, glucose values were not available in 13 women, leaving 971 women for the analysis. Detailed maternal anamnestic and sociodemographic factors are shown in Table 1. Changing maternal characteristics and OGTT results at the different periods of pregnancy studied are described in Table 2 . Figure 1 shows the flux diagram for recruitment and inclusion at each pregnancy period.

\section{GDM/overt diabetes prevalence}

At $\leq 19+6$ weeks $27.9 \%$ women were diagnosed with diabetes (27\% GDM and $0.9 \%$ overt diabetes). Glycemia leading to diagnosis was FPG in most cases (81.2\%).

At 24-28 weeks of pregnancy, diabetes was diagnosed in $15.5 \%$ of 580 previously glucose-tolerant participants (15.3\% GDM and $0.2 \%$ overt diabetes). Diabetes was diagnosed in $57.8 \%$ of the participants by FPG.

At $35-37$ weeks, diabetes was diagnosed in $15.2 \%$ of 422 previously glucose-tolerant women (15.2\% GDM). GDM was diagnosed in $42.2 \%$ by FPG.

Overall, 425 out of 971 women with glucose data (43.8\%) presented GDM/overt diabetes. In women completely adhering to the study protocol, the prevalence of GDM/overt diabetes was 52.4\% (425 out of 811 participants).

\section{GDM/overt diabetes risk factors in different periods of pregnancy and OGTT time points}

At $\leq 19+6$ weeks of gestation

At this assessment, previous AGT, previous GDM, NC, RHR and recruitment site were significantly associated with GDM/overt diabetes in the multivariate logistic regression analysis (Table 3). The direction of the association was positive for all risk factors with the exception of recruitment site with ORs ranging from 0.12 to 0.83 .

Among factors associated with overall GDM/overt diabetes, different risk factors were associated with glucose

Table 1 Prepregnancy and pregnancy characteristics of women recruited to participate in the DALI study.

\begin{tabular}{|c|c|}
\hline Characteristic & $\begin{array}{c}\text { Values, } \%, \\
\text { mean }( \pm \text { s.D.) or } \\
\text { median (IQR) }\end{array}$ \\
\hline \multicolumn{2}{|l|}{ Family history } \\
\hline First-degree relative with diabetes $(\mathrm{Y})$ & 24.6 \\
\hline \multicolumn{2}{|l|}{$\begin{array}{l}\text { Age, ethnicity and socioeconomic } \\
\text { characteristics }\end{array}$} \\
\hline Maternal age (years) & $32.1( \pm 5.3)$ \\
\hline Ethnicity (Caucasian) (Y) & 86.4 \\
\hline Education (high) (Y) & 55.1 \\
\hline Marital status (with partner) $(\mathrm{Y})$ & 93.5 \\
\hline Working status (Y) & 78.3 \\
\hline \multicolumn{2}{|l|}{ Smoking and clinical conditions } \\
\hline$P \operatorname{COS}(Y)$ & 10.3 \\
\hline Chronic hypertension (Y) & 12.5 \\
\hline Active smoking $(\mathrm{Y})$ & 16.6 \\
\hline $\begin{array}{l}\text { Previous abnormal glucose } \\
\text { tolerance }(Y)\end{array}$ & 4.0 \\
\hline \multicolumn{2}{|l|}{ Obstetric history } \\
\hline Previous pregnancies $(\mathrm{Y})$ & 62.7 \\
\hline $\begin{array}{l}\text { Previous gestational diabetes } \\
\text { mellitus }(\mathrm{Y})\end{array}$ & 6.5 \\
\hline Previous stillbirth $(\mathrm{Y})$ & 7.1 \\
\hline Previous congenital malformations $(\mathrm{Y})$ & 2.5 \\
\hline Previous macrosomia $(\mathrm{Y})$ & 13.3 \\
\hline \multicolumn{2}{|l|}{ Anthropometrics } \\
\hline Height $(\mathrm{cm})$ & $166(161-170)$ \\
\hline Prepregnancy weight (kg) & $83.5(91-100)$ \\
\hline Prepregnancy BMI $\left(\mathrm{kg} / \mathrm{m}^{2}\right)$ & $33.0(30.5-36.3)$ \\
\hline \multicolumn{2}{|l|}{$\begin{array}{l}\text { Current pregnancy-related variables } \\
\quad(\% \text { participants })\end{array}$} \\
\hline \multicolumn{2}{|l|}{ Site $(Y)$} \\
\hline Spain & 9.5 \\
\hline Austria & 11.0 \\
\hline Belgium & 9.7 \\
\hline Denmark (Copenhagen, Odense) & 20.3 \\
\hline Poland & 9.9 \\
\hline Italy (Padua, Pisa) & 10.8 \\
\hline Ireland & 7.9 \\
\hline The Netherlands & 8.0 \\
\hline The United Kingdom & 11.9 \\
\hline Male newborn sex (male) (Y) & 51.5 \\
\hline
\end{tabular}

IQR, interquartile range; PCOS, polycystic ovarian syndrome; Y, Yes. 
Table 2 Maternal characteristics and OGTT results at different periods of pregnancy. Values are expressed in $\%$, mean \pm S.D. or P50 (P25-P75)

\begin{tabular}{l}
\hline Characteristic \\
\hline Weight $(\mathrm{kg})$ \\
BMI $\left(\mathrm{kg} / \mathrm{m}^{2}\right)$ \\
Neck circumference $(\mathrm{cm})$ \\
Resting heart rate $(\mathrm{bpm})$ \\
Weight gain $(\mathrm{kg})^{\dagger}$ \\
BMI gain $\left(\mathrm{kg} / \mathrm{m}^{2}\right)^{\dagger}$ \\
Active smoking $(\mathrm{Y})$ \\
Season when OGTT was performed $(\mathrm{Y})$ \\
Spring \\
Summer \\
Autumn \\
Winter \\
DALI intervention $(\mathrm{Y})^{\ddagger}$ \\
Healthy eating \\
Physical activity \\
Vitamin D \\
Control \\
Plasma glucose concentrations (mmol/L) \\
Fasting glucose \\
1-h glucose \\
2-h glucose \\
GDM/overt diabetes new diagnosis (Y) \\
GDM/overt diabetes diagnosis by OGTT \\
time point (\%) \\
Fasting glucose \\
1-h glucose \\
2-h glucose \\
\hline
\end{tabular}

\begin{tabular}{c}
\hline $\mathbf{1 9 + 6}$ weeks $(n=971)^{*}$ \\
\hline $94.0(85.4-103.2)$ \\
$33.8(31.7-37.0)$ \\
$36.2(35.0-37.9)$ \\
$80.0(73-87)$ \\
$1.8(-0.4$ to 4.2$)$ \\
$0.64(-0.14$ to 1.55$)$ \\
16.6
\end{tabular}

\begin{tabular}{c}
\hline 24-28 weeks $(n=580)^{*}$ \\
\hline $96.4(89.5-105.4)$ \\
$34.9(33.0-37.9)$ \\
$36.0(34.9-37.9)$ \\
$84.0(77-91)$ \\
$5.6(2.2-9.1)$ \\
$1.95(0.79-3.40)$
\end{tabular}

28.3

28.2

24.4

19.2

$4.7(4.4-5)$
$7.0(5.9-8.3)$
$6.0(5.2-6.9)$
27.9

81.2
12.9
5.9

\begin{tabular}{c}
\hline 35-37 weeks $(n=422)^{*}$ \\
\hline $100.4(92.8-110.3)$ \\
$36.6(34.1-39.4)$ \\
$36.5(35.2-38.0)$ \\
$87.0(79.0-93.0)$ \\
$9.2(5.1-13.7)$ \\
$3.36(1.87-4.96)$ \\
12.3
\end{tabular}

12.3

25.2

23.6

30.5

20.8

52.6

50.9

10.9

21.1

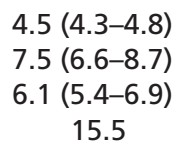

$4.4(4.2-4.7)$

$8.0(7.0-8.9)$

$6.3(5.6-7.2)$

15.2

${ }^{*} n$ only includes women with available glucose values at first assessment and without a previous GDM/overt diabetes diagnosis afterwards; ${ }^{\dagger}$ used in the sensitivity analysis instead of weight and BMl; ${ }^{\ddagger}$ percentages sum more than $100 \%$ because of combined interventions.

GDM, gestational diabetes mellitus; OGTT, oral glucose tolerance test; $Y$, yes.

values over the cut-off at different OGTT time points (Table 3). That is, NC and recruitment site were associated with abnormal FPG but not with postchallenge PG, RHR with abnormal postchallenge PG, but not with FPG, while previous AGT was associated with both abnormal fasting and postchallenge PG (Table 3).

The ORs for GDM/overt diabetes of continuous variables turned into tertiles were for NC, 0.92 (95\% CI: $0.61-1.39, P=0.68)$, for the second tertile $(35.4-37.2 \mathrm{~cm}$, $n=326)$ and 1.58 (95\% CI: $1.06-2.36, P=0.02)$ for the upper tertile $(>37.2 \mathrm{~cm}, n=315)$; and for RHR, 1.49 (95\% CI: $1.00-2.23, P=0.05$ ) for the second tertile (75 to $84 \mathrm{bpm}, n=318$ ) and 1.99 (95\% CI: $1.31-3.00, P=0.001$ ) for the upper tertile ( $>84 \mathrm{bpm}, n=293$ ).

The AUROC for the model at $\leq 19+6$ weeks of pregnancy was 0.74 (95\% CI: $0.70-0.77, P<0.001)$ (Fig. 2).

\section{At 24-28 weeks of gestation}

In the multivariate analysis, stillbirth in a prior pregnancy, RHR and recruitment site were the independent variables associated with GDM/overt diabetes (Table 3). The direction of the association was positive for unexpected stillbirth and RHR but not for recruitment site (OR: 0.09-0.78).

Among factors associated with both overall GDM/overt diabetes and abnormal PG at specific OGTT time points, stillbirth in a prior pregnancy was associated with postchallenge PG, while RHR was associated with both abnormal fasting and postchallenge PG (Table 3). The ORs for GDM/overt diabetes for tertiles of RHR were 1.83 (95\% CI: $0.93-3.60, P=0.08$ ), for the second tertile (80-88bpm, $n=184)$ and 3.32 (95\% CI: 1.70-6.49, $P<0.001)$ for the upper tertile ( $>88 \mathrm{bpm}, n=182$ ).

The AUROC for the model between 24 and 28 weeks of pregnancy was 0.71 (95\% CI: $0.65-0.78, P<0.001$ ) (Fig. 2).

\section{At 35-37 weeks of gestation}

At this gestational age, only maternal height was (negatively) associated with GDM/overt diabetes in the multivariate logistic regression analysis (Table 3) but did 


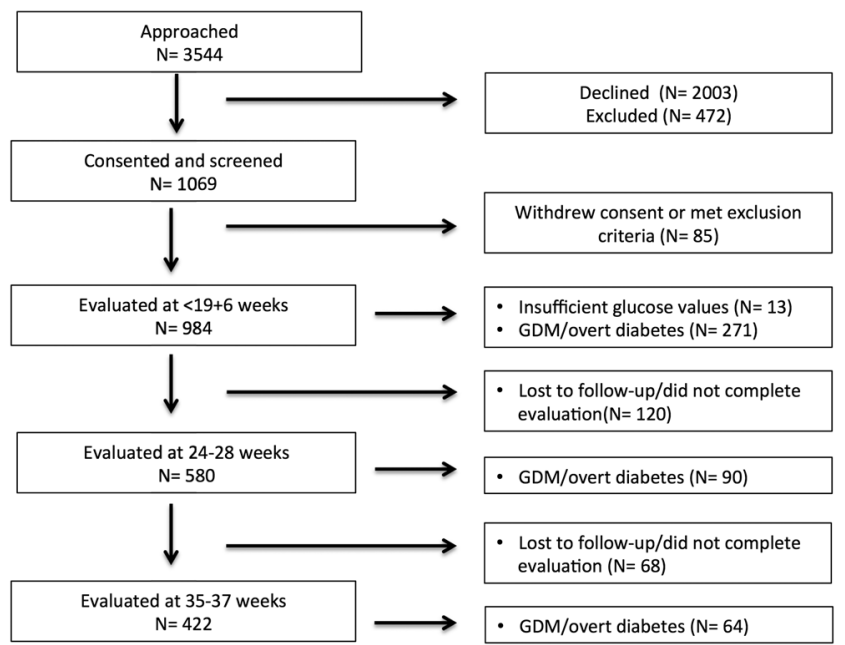

Figure 1

Flux diagram for recruitment and inclusion at different pregnancy periods. GDM, gestational diabetes mellitus.

not achieve significance for the individual time points. The ORs for GDM/overt diabetes for tertiles of maternal height were 0.99 (95\% CI: $0.54-1.83, P=0.98$ ) for the second tertile $(163-169 \mathrm{~cm}, n=200$ and 0.41 (95\% CI: 0.20-0.87, $P=0.02)$ ) for the upper tertile ( $>169 \mathrm{~cm}, n=195)$.

The AUROC for the model between 35 and 37 weeks of pregnancy was 0.60 (95\% CI: $0.53-0.68, P=0.009$ ) (Fig. 2).

\section{Sensitivity analysis}

In the first sensitivity analysis, $\triangle \mathrm{BMI}$ was added to the models for abnormal FPG and GDM/overt diabetes after any OGTT time point at 24-28 weeks of pregnancy (details not shown with a nominal improvement in the AUROC for this period (AUROC $=0.726,95 \% \mathrm{CI}$ : 0.667-0.785).

In the second sensitivity analysis, the mixed-effects logistic regression showed that center continue to be significant both at $<19+6$ and $24-28$ weeks and the magnitude of the effect for other independent variables was similar.

\section{Discussion}

Overweight and obesity are major risk factors for GDM and their prevalence among women of reproductive age is on the rise. Identifying risk factors for GDM in earlier or later periods of pregnancy could help optimize screening strategies and interventions.
In this analysis of the DALI study, we report that different clinical risk factors are associated with GDM/ overt diabetes at different pregnancy periods and OGTT time points. At first assessment previous GDM, previous AGT and NC were associated with GDM/overt diabetes, alongside recruitment site and RHR. The last two variables, as well as previous stillbirth were also associated with GDM/overt diabetes at 24-28 weeks, whereas in late pregnancy, only maternal height was identified. As for the different OGTT time points, we observed that NC and recruitment site were associated with FPG, stillbirth with abnormal postchallenge PG and prior AGT and RHR with both abnormal FPG and postchallenge PG.

\section{GDM/overt diabetes prevalence}

The prevalence of GDM/overt diabetes in the participants of the DALI study was high (43.8\%), information that has already been reported $(17,18)$. The variation in the figures herein described is attributable to the use of central lab values in this report.

\section{Clinical risk factors associated with GDM/overt diabetes after any OGTT time point in different periods of pregnancy}

The DALI study has published two reports where risk factors for GDM have been addressed $(17,18)$ but not specifically in the three study pregnancy periods and OGTT time points as in the current study.

At $\leq 19+6$ weeks, the identified risk factors for GDM/ overt diabetes were previous AGT, previous GDM, NC, RHR and recruitment site. All these variables have been reported in the literature as associated with overall GDM (diagnosed at any time during pregnancy).

The association of previous GDM with GDM diagnosed before 24 weeks of pregnancy has been described (19), and it is the only risk factor considered by the current NICE guidelines to qualify for GDM testing in early pregnancy (7).

We are not aware of studies addressing NC, previous AGT, RHR or recruitment site at first assessment.

Central adiposity and specifically NC has been reported to confer additional risk for AGT beyond BMI both in (20) and outside pregnancy (10). In the UPBEAT trial, neck/ thigh ratio superseded BMI, as an independent variable for GDM at 27-28 weeks (21). Our study confirms that NC out-performs BMI itself as risk factor for GDM/overt diabetes, in overweight/obese women and shows for the first time that this association is present at $\leq 19+6$ weeks. 


,

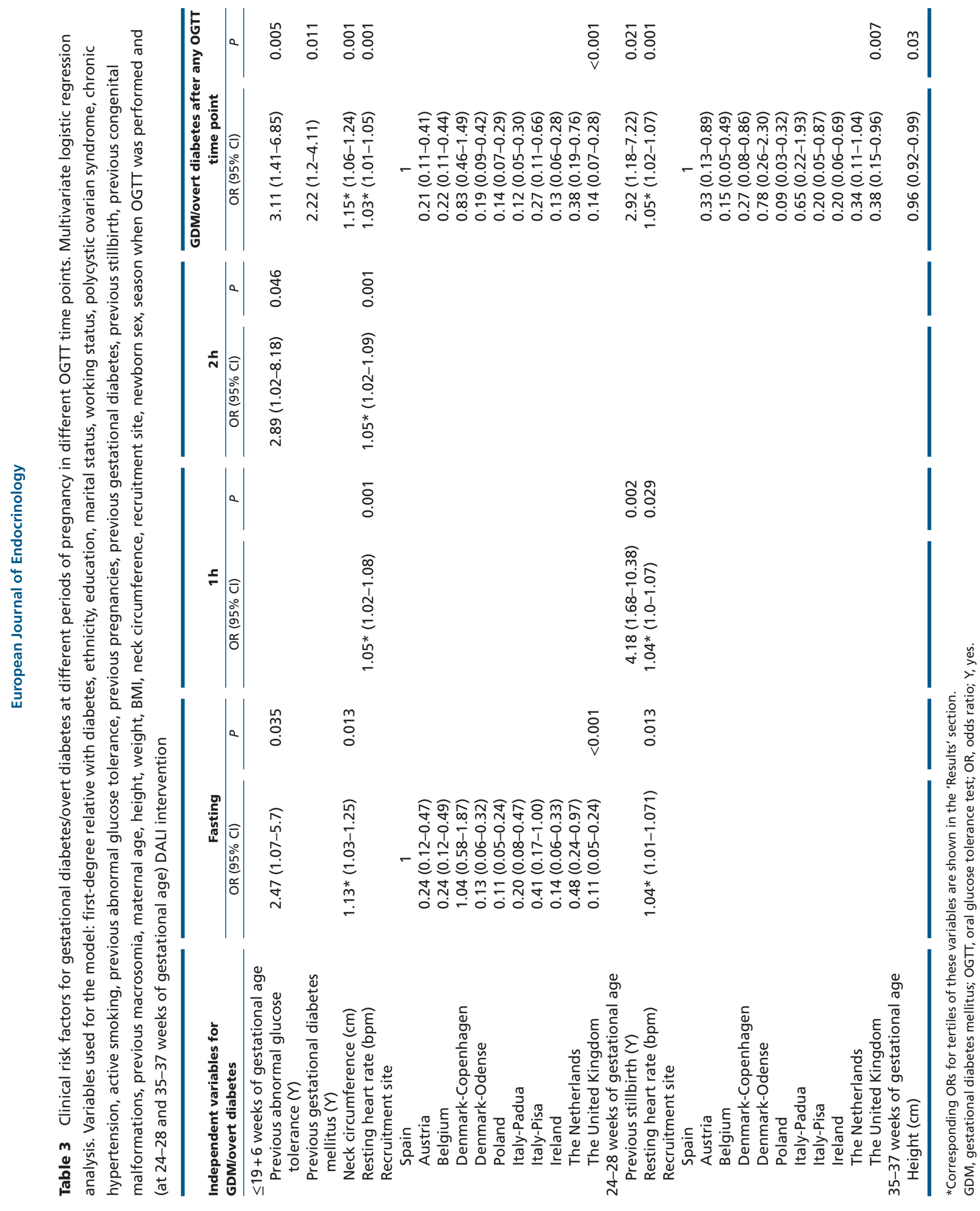



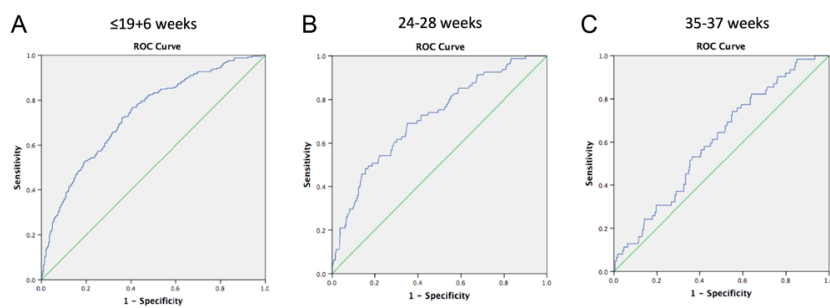

\section{Figure 2}

Receiver-operating characteristic curves for GDM/overt DM models after clinical factors with assessment of maternal size using measurements during pregnancy. (A) at $\leq 19+6$ weeks of gestational age, (B) between 24 and 28 weeks, (C) and between 35 and 37 weeks. GDM, gestational diabetes mellitus; ROC, receiver-operating characteristic curve.

RHR has been validated as a proxy for fitness status (22) but has not been directly addressed as a risk factor for GDM. We herein report for the first time that increasing RHR is a risk factor for GDM/overt diabetes at $<19+6$ weeks. According to the observed ORs expressed for tertiles of RHR and NC, RHR has a larger contribution for identifying GDM/overt diabetes at this gestational age.

A large variation in the prevalence of diabetes among different locations is widely known, both in and outside pregnancy $(5,23,24)$. In the current analysis, we have observed an eight-fold difference in the risk of GDM/overt diabetes between centers, but it did not fit the previously reported Atlantic-to-Mediterranean gradient in prevalence (24). However, we have to take into account that the Atlantic-to-Mediterranean gradient referred to absolute prevalence. Even more intriguing, different sites of the same country (i.e. Copenhagen vs Odense in Denmark) displayed different ORs for GDM/overt diabetes. Genetic, environmental and/or lifestyle factors such as diet or pollution could be responsible for these differences.

Although the use of IADPSG criteria to diagnose GDM before 24-28 weeks has recently been challenged due to the physiological decrease of FPG from early pregnancy to 24-28 weeks, this is not the case for obese women (25). Thus, we assume that if we had not used IADPSG criteria at first assessment, risk factors for GDM at $\leq 19+6$ would translate into risk factors at 24-28 weeks.

At 24-28 weeks of pregnancy, the independent variables for GDM/overt diabetes were previous stillbirth, RHR and recruitment site.

The current observation of prior stillbirth conferring a $\approx$ three-fold higher risk of GDM/overt diabetes at 24-28 weeks is in line with information in the literature reporting unexplained stillbirth to be a risk factor for AGT/GDM in a subsequent pregnancy (OR: 4.52) (26).

PA has been linked with a lower risk of GDM (27) while exercise intervention trials have shown differing impact in GDM prevalence $(28,29)$. In the DALI study itself, the PA group showed no improvement in the rate of GDM (30). As the PA intervention did not have an impact on RHR at 24-28 or 35-37 weeks (data not shown), this could explain the lack of benefit of PA intervention on metabolic outcomes.

The discussion on recruitment site as a risk factor for GDM/overt diabetes at $\leq 19+6$ weeks would also apply here.

At 35-37 weeks of gestational age, maternal height, a non-modifiable risk factor, was the only independent variable associated with GDM/overt diabetes. Maternal height is also a known risk factor for GDM, showing an inverse association (31). As to the underlying mechanisms, components of stature, specifically leg length, are inversely associated with insulin secretion and sensitivity (32). The relevance of identifying risk factors for GDM/ overt diabetes at 35-37 weeks is unclear as the impact of hyperglycemia diagnosed at this stage is unknown.

\section{Sensitivity analysis}

In the sensitivity analysis $\Delta$ BMI was an independent variable for GDM/overt diabetes at 24-28 weeks. This indicates the contribution of $\Delta$ weight during pregnancy and the potential to address it for GDM prevention despite the fact that this approach has not been successful in DALI among other trials (33).

\section{Clinical risk factors for abnormal PG at different OGTT time points}

The relevance of abnormal glucose levels at different OGTT time points comes from their different impact on outcomes, from their relative contribution to the GDM diagnosis itself (3), and for different pathophysiology since subjects with impaired FPG display basal insulin resistance $(34,35)$ while subjects with impaired glucose tolerance display impaired insulin secretion (34).

In pregnancy, a single study showed that FPG was associated with maternal weight, $1 \mathrm{~h}$ PG was associated with maternal weight, height, parity and socioeconomic condition, while no associations with $2 \mathrm{~h}$ PG were identified (35). In our study, different methods and covariates were used but both studies concur in that fasting and postchallenge PGs are associated with different variables. 
We have observed that NC was associated with abnormal FPG at $\leq 19+6$ weeks of pregnancy. In nonpregnant women, NC is associated with FPG (10) but in pregnancy, its relationship with specific OGTT time points had not been previously described.

Previous AGT was associated with $\mathrm{a} \approx$ two-fold risk of abnormal FPG and $\approx$ three-fold risk of abnormal $2 \mathrm{~h}$ PG in the first assessment, which is not surprising, considering that the definition of AGT includes both impaired FPG and postchallenge PG.

RHR was associated with abnormal postchallenge PG at $<19+6$, and with both fasting and postchallenge PG at 24-28 weeks. Measures of cardiorespiratory fitness are mainly associated with postchallenge PG both outside and during pregnancy $(29,36)$. Our results indicate that in pregnant women, surrogates of exercise also display favorable associations with FPG but preferentially with postchallenge PG.

Recruitment site was an independent variable for GDM/overt diabetes at first assessment due to its association with abnormal FPG. This is in line with the HAPO study, where adjusting for BMI did not eliminate differences between sites in abnormal FPG suggesting that other unknown site-related factors had an impact on FPG (5).

At 24-28 weeks of gestation, previous stillbirth was associated with abnormal 1-h PG. This would be in agreement with the post hoc analysis of the aspart trial in women with type $1 \mathrm{DM}$ where spikes of high glucose values in the third trimester were associated with poor late pregnancy outcomes including stillbirth (37).

Thus, we have identified that distinct clinical characteristics are associated with abnormal PG at different OGTT time points.

There is a growing interest to identify women who will develop GDM in order to either use selective screening approaches or to test prevention strategies. In high-risk populations, AUROCs as high as 0.88 have been reported for early GDM (19) and 0.82 for GDM at 24-28 weeks (38). In our opinion, the AUROC curves observed in the current report (0.74 at first assessment and 0.71 at 24-28 weeks) do not support selective screening among an overweight/ obese population at any of these periods. The study was conducted in a group of overweight/obese women; thus, we have not addressed the use of overweight/obesity vs normal weight as a risk factor for GDM/overt diabetes.

However, observations from the current study can help identify women at risk as well as modifiable risk factors for GDM. The association between NC and RHR (adiposity and fitness proxies), with GDM/overt diabetes diagnosed before 20 weeks of gestation indicate, that in overweight/ obese women, lifestyle changes including diet and exercise, may need to be implemented before conception to have a positive impact in metabolic outcomes at these gestational age. Clinical risk factors for GDM/overt diabetes in women not diagnosed at first assessment may help to identify a target population for preventive interventions during pregnancy and even the intervention itself (i.e. safe exercise aiming at reducing RHR).

The main strength of this study is the universal GDM/ overt diabetes screening at different pregnancy periods unless earlier diagnosis had been performed, which allows to identify risk factors for real incident GDM cases. The study population size and the multicenter setting are additional strengths being representative of the European overweight/obese population. The use at first assessment of IADPSG criteria, currently challenged, can be considered a weakness but right now there are no validated criteria for this gestational age. Self-reported data on prepregnancy weight and previous stillbirth could be considered additional weaknesses. The study was conducted in a group of overweight/obese women, which limits the results interpretation to this specific population. It could be argued that the DALI interventions could have influenced the risk factors identified. However, this is unlikely, since DALI interventions were included as potential independent variables in our analysis.

Overall, in women with a prepregnancy BMI $\geq 29 \mathrm{~kg} /$ $\mathrm{m}^{2}$, the prevalence of GDM/overt diabetes after IADPSG/ WHO2013 was very high, most associated clinical characteristics were non-modifiable, differed by pregnancy period and OGTT time point, and the AUROCs did not support selective screening. The identified clinical risk factors can help define the target population for future intervention trials with RHR making a new attractive candidate for both identification and intervention.

\section{Declaration of interest}

The authors declare that there is no conflict of interest that could be perceived as prejudicing the impartiality of this study.

\section{Funding}

This project received funding from the European Community's 7th Framework Program (FP7/2007-2013; grant agreement no. 242187). In the Netherlands, additional funding was provided by the Netherlands Organization for Health Research and Development (ZonMW) (Grant $\mathrm{nr}$ 200310013). In the UK, the DALI team acknowledges the support received from the NIHR Clinical Research Network: Eastern, especially the local diabetes clinical and research teams based in Cambridge. In Spain, additional funding was provided by CAIBER 1527-B-226. The funders had no role in any aspect of the study beyond 501 funding. 


\section{Author's contribution statement}

L C M, J H and R C made substantial contributions to project components, including study concept and design, acquisition, analysis and interpretation of data and paper drafting. All other authors contributed to acquisition and interpretation of data and have made critical intellectual contributions to the manuscript and have approved the final version.

\section{References}

1 American Diabetes Association. Standards of medical care in diabetes - 2017. Diabetes Care 201740 (Supplement) S1-S142.

2 Black MH, Sacks DA, Xiang AH \& Lawrence JM. Clinical outcomes of pregnancies complicated by mild gestational diabetes mellitus differ by combinations of abnormal oral glucose tolerance test values. Diabetes Care 201033 2524-2530. (https://doi.org/10.2337/dc10-1445)

3 Metzger BE, Lowe LP, Dyer AR, Trimble ER, Chaovarindr U, Coustan DR, Hadden DR, McCance DR, Hod M, McIntyre HD et al. Hyperglycemia and adverse pregnancy outcomes. New England Journal of Medicine 2008358 1991-2002. (https://doi.org/10.1056/ NEJMoa0707943)

4 International Association of Diabetes and Pregnancy Study Groups Consensus Panel, Metzger BE, Gabbe SG, Persson B, Buchanan TA, Catalano PA, Damm P, Dyer AR, Leiva Ad, Hod M et al. International association of diabetes and pregnancy study groups recommendations on the diagnosis and classification of hyperglycemia in pregnancy. Diabetes Care 201033 676-682. (https://doi.org/10.2337/dc09-1848)

5 Sacks DA, Coustan DR, Hadden DR, Hod M, Maresh M, Oats JJN, Deerochanawong C, Dyer AR, Metzger BE, Lowe LP et al. Frequency of gestational diabetes mellitus at collaborating centers based on IADPSG consensus panel-recommended criteria: the Hyperglycemia and Adverse Pregnancy Outcome (HAPO) study. Diabetes Care 2012 35 526-528. (https://doi.org/10.2337/dc11-1641)

6 Acosta D, Balsells M, Ballesteros M, Bandres MO, Bartha JL, Bellart J et al. Asistencia a la gestante con diabetes. Guía de práctica clínica actualizada en 2014. Avances en Diabetología 201531 45-59. (https:// doi.org/10.1016/j.avdiab.2014.12.001)

7 NICE. Diabetes in pregnancy: management of diabetes and its complications from preconception to the postnatal period. NICE Guidel NG3. 2015 pp 1-681.

8 Chu S, Callaghan W, Kim S, Schmid C, Lau J, England L \& Dietz PM. Maternal obesity and risk of gestational diabetes mellitus. Diabetes Care 200730 2070-2076. (available from: http://www.ncbi.nlm.nih. gov/pubmed/21860284) (https://doi.org/10.2337/dc06-2559a)

9 Torloni MR, Betrán AP, Horta BL, Nakamura MU, Atallah AN, Moron AF \& Valente O. Prepregnancy BMI and the risk of gestational diabetes: a systematic review of the literature with meta-analysis: Diagnostic in Obesity and Complications. Obesity Review 200910 194-203. (https://doi.org/10.1111/j.1467-789X.2008.00541.x)

10 Preis SR, Massaro JM, Hoffmann U, D'Agostino RB, Levy D, Robins SJ, Meigs JB, Vasan RS, O'Donnell CJ \& Fox CS. Neck circumference as a novel measure of cardiometabolic risk: the Framingham heart study. Journal of Clinical Endocrinology and Metabolism 201095 3701-3710. (https://doi.org/10.1210/jc.2009-1779)

11 Yang L, Samarasinghe YP, Kane P, Amiel SA \& Aylwin SJB. Visceral adiposity is closely correlated with neck circumference and represents a significant indicator of insulin resistance in WHO grade III obesity. Clinical Endocrinology 201073 197-200. (https://doi. org/10.1111/j.1365-2265.2009.03772.x)

12 Ben-noun LL, Sohar E \& Laor A. Neck circumference as a simple screening measure for identifying overweight and obese patients. Obesity Research 20019 470-477. (https://doi.org/10.1038/oby.2001.61)

13 Ursavas A, Karadag M, Nalci N, Ercan I \& Gozu RO. Self-reported snoring, maternal obesity and neck circumference as risk factors for pregnancy-induced hypertension and preeclampsia. Respiration 2008 76 33-39. (https://doi.org/10.1159/000107735)

14 Verburg PE, Tucker G, Scheil W, Jaap J, Erwich HM, Dekker GA \& Roberts CT. Seasonality of gestational diabetes mellitus: a South Australian population study. BMJ Open Diabetes Research and Care 20164 e000286. (https://doi.org/10.1136/bmjdrc-2016-000286)

15 Retnakaran R, Kramer CK, Ye C, Kew S, Hanley AJ, Connelly PW, Sermer M \& Zinman B. Fetal sex and maternal risk of gestational diabetes mellitus: the impact of having a boy. Diabetes Care 201538 844-851. (https://doi.org/10.2337/dc14-2551)

16 Jelsma GM, Van NM, Galjaard S, Desoye G, Corcoy R, Devlieger R, van Assche A, Timmerman D, Jans G, Harreiter J et al. DALI: vitamin $\mathrm{D}$ and lifestyle intervention for gestational diabetes mellitus (GDM) prevention: an European multicentre, randomised trial - study protocol. BMC Pregnancy and Childbirth 201313 16. (https://doi. org/10.1186/1471-2393-13-16)

17 Harreiter J, Simmons D, Desoye G, Corcoy R, Adelantado JM, Devlieger R, van Assche A, Galjaard S, Damm P, Mathiesen ER et al. IADPSG and WHO 2013 gestational diabetes mellitus criteria identify obese women with marked insulin resistance in early pregnancy. Diabetes Care 201639 e90-e92. (https://doi.org/10.2337/dc16-0200)

18 Egan AM, Vellinga A, Harreiter J, Simmons D, Desoye G, Corcoy R, Adelantado JM, Devlieger R, Van Assche A, Galjaard S et al. Epidemiology of gestational diabetes mellitus according to IADPSG/ WHO 2013 criteria among obese pregnant women in Europe. Diabetologia 201760 1913-1921. (https://doi.org/10.1007/s00125-0174353-9)

19 Sweeting AN, Wong J, Appelblom H, Ross GP, Kouru H, Williams PF, Sairanen M \& Hyett JA. A first trimester prediction model for gestational diabetes utilising aneuploidy and pre-eclampsia screening markers. Journal of Maternal-Fetal and Neonatal Medicine 201831 2122-2130. (https://doi.org/10.1080/14767058.2017.1336759)

20 He F, He H, Liu W, Lin J, Chen B, Lin Y, Zhao Y, Tao W \& Xia X. Neck circumference might predict gestational diabetes mellitus in Han Chinese women: a nested case-control study. Journal of Diabetes Investigation 20178 168-173. (https://doi.org/10.1111/jdi.12574)

21 White SL, Lawlor DA, Briley AL, Godfrey KM, Nelson SM, OtengNtim E, Robson SC, Sattar N, Seed PT, Vieira MC et al. Early antenatal prediction of gestational diabetes in obese women: development of prediction tools for targeted intervention. PLOS ONE $2016 \mathbf{1 1}$ e0167846. (https://doi.org/10.1371/journal.pone.0167846)

22 Blair SN, Kannel WB, Kohl HW, Goodyear N \& Wilson PW. Surrogate measures of physical activity and physical fitness. Evidence for sedentary traits of resting tachycardia, obesity, and low vital capacity. American Journal of Epidemiology 1989129 1145-1156. (https://doi. org/10.1093/oxfordjournals.aje.a115236)

23 Hansen CN \& Borch-Johnsen K. Age, body mass index and glucose tolerance in 11 European population-based surveys. Diabetic Medicine 200219 558-565. (https://doi.org/10.1046/j.1464-5491.2002.00741.x)

24 Buckley BS, Harreiter J, Damm P, Corcoy R, Chico A, Simmons D, Vellinga A, Dunne F \& DALI Core Investigator Group. Gestational diabetes mellitus in Europe: prevalence, current screening practice and barriers to screening. A review. Diabetic Medicine 201229 844-854. (https://doi.org/10.1111/j.1464-5491.2011.03541.x)

25 Mills JL, Jovanovic L, Knopp R, Aarons J, Conley M, Park E, Lee YJ, Holmes L, Simpson JL \& Metzger B. Physiological reduction in fasting plasma glucose concentration in the first trimester of normal pregnancy: the diabetes in early pregnancy study. Metabolism 1998 47 1140-1144. (https://doi.org/10.1016/S0026-0495(98)90290-6)

26 Robson S, Chan A, Keane RJ \& Luke CG. Subsequent birth outcomes after an unexplained stillbirth: preliminary population-based retrospective cohort study. Australian and New Zealand Journal of Obstetrics and Gynaecology 200141 29-35. (https://doi.org/10.1111/ j.1479-828X.2001.tb01290.x)

27 Oken E, Ning Y, Rifas-Shiman SL, Radesky JS, Rich-Edwards JW \& Gillman MW. Associations of physical activity and inactivity 
before and during pregnancy with glucose tolerance. Obstetrics and Gynecology 2006108 1200-1207. (https://doi.org/10.1097/01. AOG.0000241088.60745.70)

28 Han S, Middleton P \& Crowther CA. Exercise for pregnant women for preventing gestational diabetes mellitus. Cochrane Database of Systematic Reviews 20127 CD009021. (https://doi. org/10.1002/14651858.CD009021.pub2)

29 Wang C, Wei Y, Zhang X, Zhang Y, Xu Q, Sun Y, Su S, Zhang L, Liu C, Feng $\mathrm{Y}$ et al. A randomized clinical trial of exercise during pregnancy to prevent gestational diabetes mellitus and improve pregnancy outcome in overweight and obese pregnant women. American Journal of Obstetrics and Gynecology 2017216 340-351. (https://doi. org/10.1016/j.ajog.2017.01.037)

30 Simmons D, Jelsma JGM, Galjaard S, Devlieger R, Van Assche A, Jans G, Corcoy R, Adelantado JM, Dunne F, Desoye G et al. Results from a European multicenter randomized trial of physical activity and/or healthy eating to reduce the risk of gestational diabetes mellitus: The DALI lifestyle pilot. Diabetes Care 201538 1650-1656. (https://doi.org/10.2337/dc15-0360)

31 Anastasiou E, Alevizaki M, Grigorakis SJ, Philippou G, Kyprianou M \& Souvatzoglou A. Decreased stature in gestational diabetes mellitus. Diabetologia 199841 997-1001. (https://doi.org/10.1007/s001250051022)

32 Vangipurapu J, Stančáková A, Jauhiainen R, Kuusisto J \& Laakso M. Short adult stature predicts impaired beta-cell function, insulin resistance, glycemia and type 2 diabetes in Finnish men. Journal of Clinical Endocrinology and Metabolism 2016102 443-450. (https://doi. org/10.1210/jc.2016-2933)

33 Simmons D, Devlieger R, Van Assche A, Jans G, Galjaard S, Corcoy R, Adelantado JM, Dunne F, Desoye G, Harreiter J et al. Effect of physical activity and/or healthy eating on GDM risk: the DALI lifestyle study. Journal of Clinical Endocrinology and Metabolism 2017 102 903-913. (https://doi.org/10.1210/jc.2016-3455)

34 Tripathy D, Carlsson M, Almgren P, Isomaa B, Taskinen MR, Tuomi T \& Groop LC. Insulin secretion and insulin sensitivity in relation to glucose tolerance: lessons from the Botnia Study. Diabetes 200049 975-980. (https://doi.org/10.2337/diabetes.49.6.975)

35 Gopalakrishnan V, Singh R, Pradeep Y, Kapoor D, Rani A, Pradhan S, Bhatia E \& Yadav SB. Evaluation of the prevalence of gestational diabetes mellitus in North Indians using the International Association of Diabetes and Pregnancy Study groups (IADPSG) criteria. Journal of Postgraduate Medicine 201561 155-158. (https:// doi.org/10.4103/0022-3859.159306)

36 Bonnet F, Empana JP, Natali A, Monti L, Golay A, Lalic K, Dekker J, Mari A, Balkau B \& RISC Study Group. Elevated heart rate predicts $\beta$ cell function in non-diabetic individuals: the RISC cohort. European Journal of Endocrinology 2015173 409-415. (https://doi.org/10.1530/ EJE-15-0115)

37 Damm P, Mersebach H, Råstam J, Kaaja R, Hod M, McCance DR $\&$ Mathiesen ER. Poor pregnancy outcome in women with type 1 diabetes is predicted by elevated $\mathrm{HbA}_{1 \mathrm{c}}$ and spikes of high glucose values in the third trimester. Journal of Maternal-Fetal and Neonatal Medicine 201427 149-154. (https://doi.org/10.3109/14767058.2013. 806896)

38 Savvidou M, Nelson SM, Makgoba M \& Messow C. First-trimester prediction of gestational diabetes mellitus : examining the potential of combining maternal characteristics and laboratory measures. Diabetes 201059 3017-3022. (https://doi.org/10.2337/ db10-0688)

Received 4 January 2018

Revised version received 16 April 2018

Accepted 8 May 2018 